\begin{tabular}{|c|c|c|c|c|c|}
\hline & & $\begin{array}{l}\text { NTproBNP to } \\
\text { referral (days) }\end{array}$ & & $\begin{array}{l}\text { Referral to clinic } \\
\text { review (days) }\end{array}$ & \\
\hline & $\begin{array}{l}\text { Number of } \\
\text { Patients (n) }\end{array}$ & $\begin{array}{l}\text { Mean } \pm \text { Standard } \\
\text { Deviation }\end{array}$ & Range & $\begin{array}{l}\text { Mean } \pm \text { Standard } \\
\text { Deviation }\end{array}$ & Range \\
\hline $\begin{array}{l}2 \text { week } \\
\text { pathway }\end{array}$ & 211 & $16 \pm 16$ & $\begin{array}{l}-0- \\
124\end{array}$ & $11 \pm 7$ & $1-20$ \\
\hline $\begin{array}{l}6 \text { week } \\
\text { pathway }\end{array}$ & 209 & $19 \pm 19$ & $\begin{array}{l}-4- \\
188\end{array}$ & $34 \pm 1$ & $4-42$ \\
\hline
\end{tabular}

therefore recommend that patients with suspected HF and raised natriuretic peptides are seen for echocardiography and specialist assessment within 2 or 6 weeks of referral. We established a dedicated HF clinic locally; natriuretic peptides are checked in primary care and patients with a positive result are referred via fax. Given the time-critical nature of these guidelines we investigated local adherence to the 2 and 6week targets and the interval from a positive natriuretic result in primary care to referral.

Methods Information on the referral pathway was disseminated to all primary care referrers. Patient data were prospectively collected after establishment of the HF clinic in February 2014 to the end of November 2015. The interval from a positive NTproBNP result to referral and the interval from referral to appointment were calculated. Basic demographic data, NTproBNP level and the final specialist opinion as to whether the patient had heart failure were also collated. Patients with incomplete data sets were excluded.

Results A total of 432 patients were included. 226 (52\%) were female and 206 (48\%) male. Mean age was $78 \pm 9$ and mean NTproBNP $3101 \pm 4445$ pg/ml. 239 (55\%) patients had a diagnosis of HF following review, of these $123(51.5 \%)$ had a reduced ejection fraction and 116 (48.5\%) a preserved ejection fraction.

All $212(100 \%)$ patients were offered an appointment within the 6 week target and 211 (97\%) within the 2 week target. 9 patients (4\%) in the 2 week pathway and $3(1 \%)$ in the 6 week pathway declined the appointment that was offered and where therefore seen beyond the target timeframe.

There were significant delays between the positive NTproBNP result and referral to the HF clinic (Table 1). Most notably for the 2 week pathway the mean interval from a positive NTproBNP to referral was longer than the interval from referral to appointment (16 days versus 11 days respectively). One patient was referred 124 days after the positive NTproBNP result.

Conclusion The purpose of including referral-time targets in the NICE guidelines is so that patients with suspected HF are seen rapidly as they are at high risk of adverse outcome. Despite offering a service compliant with these targets we've identified significant delays between a positive NTproBNP result in primary care and referral to clinic. This unnecessary delay may have an adverse effect on outcome. Further refinement of the primary care aspect of the referral pathway is required.

\section{NT PRO-BNP TEST CAN SAVE NHS $£$ 1.6 MILLION PER YEAR}

${ }^{1}$ Chetan Upadhyaya*, ${ }^{2}$ Kathyrn Roberts, ${ }^{2}$ Geraint Jenkins, ${ }^{2}$ Aaron Wong. 'West Wales General Hospital; ${ }^{2} A B M U$ HB; * Presenting Author

\subsection{6/heartjnl-2016-309890.15}

Purpose To find out the number of inappropriate echocardiogram requests made despite a negative NT pro-BNP test $(<400 \mathrm{pg} / \mathrm{l})$ and in even more negative test $(<300 \mathrm{pg} / \mathrm{l})$.

To estimate the cost burden on NHS.

To look at the impact on waiting times in the department from these requests.

Method We conducted a retrospective audit reviewing the NT pro-BNP requests in

Abertawe BroMorgannwg University Health Board. Data was collected from all NT pro-BNP assays from January 2013 to January 2014. The criteria of NT pro-BNP level of less than $400 \mathrm{ng} / \mathrm{l}$ was set according to the NICE Guidelines for Chronic heart failure 2010. We also looked if any requests were made when BNP levels were less than $300 \mathrm{ng} / \mathrm{l}$.

Findings 370 NT pro-BNP requests were done during this period and $334(90.27 \%)$ requests were from primary care. 300 assays were less than $400 \mathrm{ng} / \mathrm{l}$ and essentially ruled out heart failure. Regardless of the results, 190 requests were made for an echocardiogram to assess Left Ventricular function. 137 out of $190(72 \%)$ of these echo requests were made in patients with a BNP level of less than $400 \mathrm{ng} / \mathrm{l}$. The mean BNP level in patients with values of less than $400 \mathrm{ng} / \mathrm{l}$ was $120 \mathrm{ng} / \mathrm{l}$. 278/370 assays had a BNP value of $<300 \mathrm{pg} / \mathrm{l}$ making heart failure very unlikely, but 120 echo requests were made in this group.

Conclusion Despite high negative predictive value of NT proBNP levels, significant amount of echo requests are made. The burden on our echo department is an extra 137 requests which amounts to 34.5 sessions by a sonographer/year. This amounts to 6,400 wasted echo sessions in NHS/year. The financial burden to NHS is estimated at around 137 inappropriate echo requests in 160 NHS Foundation Trust/Health Board at a cost of $£ 75 /$ scan $=£ 1.6$ million/year.

Recommendation Health professionals requesting the above tests should be made aware of the above findings and educated especially in the primary care setting.

The echo department should have strict criteria to perform echocardiograms in patients with normal NT pro-BNP levels.

\section{REVIEW IN A NICE COMPLIANT SPECIALIST CLINIC IS ASSOCIATED WITH REDUCED HOSPITALISATION IN PATIENTS WITH SUSPECTED HEART FAILURE}

${ }^{1}$ Legate Philip*, ${ }^{2}$ Paul Kalra, ${ }^{2}$ Geraint Morton. ${ }^{1}$ NHS; ${ }^{2}$ NHS Hospital; *Presenting Author

\subsection{6/heartjnl-2016-309890.16}

Introduction NICE guidelines recommend that patients with suspected heart failure (HF) and raised natriuretic peptides are referred for timely echocardiography and specialist assessment. Limited data are available on whether this affects outcome. We provide Trust-based NTproBNP assays for primary care, a simple referral pathway and a dedicated one-stop HF clinic fully compliant with NICE waiting time targets. Despite this, it was apparent that many patients with suspected $\mathrm{HF}$ and raised NTproBNP levels were not being referred and were being managed in non-specialist primary care settings. The aim 


\begin{tabular}{|c|c|c|c|}
\hline \multicolumn{4}{|c|}{ Abstract 16 Table 1} \\
\hline & HF CUINIC ( $\mathrm{n}=161)$ & NO HF CUINIC ( $\mathrm{n}=406)$ & $p$ value \\
\hline Age & $78 \pm 9$ & $80 \pm 9$ & 0.02 \\
\hline Average NTproBNP & 3352 & 2365 & $<0.0001$ \\
\hline $\begin{array}{l}\text { All-cause } \\
\text { hospitalisation }\end{array}$ & $37(22.3 \%)$ & $180(44.3 \%)$ & $<0.0001$ \\
\hline CV hospitalisation & $4(2.5 \%)$ & $57 \quad(14.0 \%)$ & $<0.0001$ \\
\hline Deaths & $9(5.6 \%)$ & 33 (8.1\%) & 0.3 \\
\hline Mean hospital stay & 4.7 days & 5.6 days & 0.42 \\
\hline
\end{tabular}

of this study was to compare hospitalisation and mortality rates in patients with suspected HF and raised NTproBNP who were reviewed in a HF clinic to those who were not referred.

Methods Data were collected retrospectively from hospital records. All patients with a raised NTproBNP (>400 pg/ml) from February to September 2014 were identified via the Trust biochemistry laboratory and were included in the study. These patients were divided into those referred to and reviewed in the clinic (HF Clinic) and those who were not referred (No HF Clinic). Hospital coding data, information services and case notes were used to identify hospital admissions, length of stay, reason for admission and mortality in all patients over a minimum follow up period of 6-months from the time of the raised NTproBNP result. Comparisons were then made between the HF clinic and No HF clinic cohorts.

Results 567 consecutive patients were identified: 161 (28\%) in the HF Clinic group and $406(72 \%)$ in the No HF Clinic group. All HF clinic patients were seen within 2 weeks (NTproBNP > $2000 \mathrm{pg} / \mathrm{ml}$ ) or 6 weeks (NTproBNP 400$2000 \mathrm{pg} / \mathrm{ml}$ ) in line with NICE recommendations. Mean follow up period was $8.9 \pm 2.3$ months. Mean patient age was $80 \pm 9$ years. The HF clinic cohort was slightly younger (Table 1). 52\% of HF clinic patients were male versus $48 \%$ of No HF clinic patients $(p=0.42$ ). Despite having a significantly higher NTproBNP level HF clinic patients had a much lower rate of all cause and cardiovascular admissions than the No HF clinic patients (Table 1 and Figure 1). There was no difference in mortality.

Conclusions Hospitalisation rates in patients with suspected heart failure and raised NTproBNP were extremely high over a relatively short follow up period. Patients reviewed in a specialist HF clinic had much higher NTproBNP levels, suggesting they were at higher risk of adverse outcomes, yet also had significantly lower rates of all cause and cardiovascular hospitalisation. The data are observational so causation and association cannot be separated however the magnitude of the difference between the patient groups suggests referral to HF clinics may improve outcomes. Our findings support implementation of the relevant NICE guidelines for patients with suspected $\mathrm{HF}$ and higher rates of referral from primary care should be encouraged.

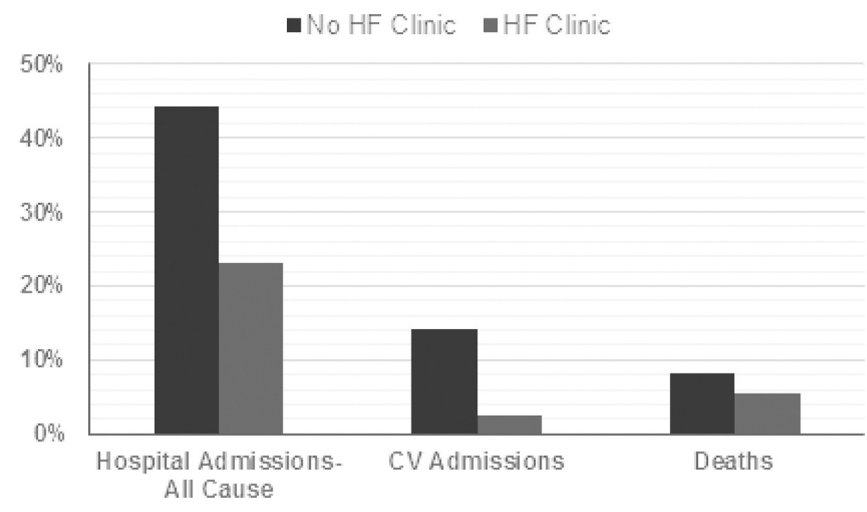

Abstract 16 Figure 1

\section{TELEMEDICINE IN PATIENTS WITH NEW DIAGNOSIS OF HEART FAILURE: FROM CLINICAL RESEARCH TO PRACTICE}

${ }^{1}$ George Koulaouzidis*, ${ }^{2}$ Kevin Mohee, ${ }^{3}$ David Barrett, ${ }^{2}$ Andrew Clark. ${ }^{1}$ Royal Brompton Hospital; ${ }^{2}$ Department of Academic Cardiology, Castle Hill Hospital; ${ }^{3}$ Faculty of Health and Social Care, University of Hull; *Presenting Author

\subsection{6/heartjnl-2016-309890.17}

Background Heart failure (HF) is a complex clinical syndrome associated with high mortality and high rate of hospital readmissions. Telehealth $(\mathrm{TH})$ is a promising strategy for improving HF outcomes but there is an urgent need to properly identify those patients in whom a TH approach would provide benefit. The purpose of this study was to determine if $\mathrm{TH}$ in patients with recently diagnosed HF and ejection fraction $<45 \%$, reduces the risk of re-admission from any cause or death from any cause.

Methods A retrospective study of 124 patients (78.2\% male; $68.6 \pm 12.6$ years; $56.5 \%$ ischaemic aetiology) who underwent $\mathrm{TH}$ and 345 patients $(68.5 \%$ male; $70.2 \pm 10.7$ years; $56.3 \%$ ischaemic aetiology) who underwent the usual-care (UC), between 2009-12. All patients had a recent diagnosis of HF, NHYA class II-III and ejection fraction $<45 \%$. The TH group were assessed by body weight, blood pressure and heart rate on a daily basis using electronic devices with automatic transfer of measured data to an online database. The followup period was 12 months.

Results Death from any cause occurred in $8.1 \%$ of the $\mathrm{TH}$ group and $19 \%$ of the UC group, $p=0.002$. Readmissions for any cause occurred in $63.7 \%$ of patients in the $\mathrm{TH}$ group and $62.5 \%$ of patients in the UC group, $p=0.8$. The number of readmissions/patients was also similar in the two groups, $1.3 \pm 1.7$ in the TH group and $1.4 \pm 1.7$ in the UC group $(p=0.9)$. A difference in the number of days in hospital was noted $(8.1 \pm 12.8$ days in $\mathrm{TH}$ group and $9.5 \pm 17.3$ in UC group) but this was not statistically significant $(p=$ $0.4)$. There was significant difference in the days alive and away from the hospital in the two groups: $348.3 \pm 5.5$ days in the TH group and $329.9 \pm 4.6$ days in the UC group ( $p$ $=0.008)$.

Conclusions In patients with a recent diagnosis of $\mathrm{HF}$ and reduced left ventricle systolic function, $\mathrm{TH}$ is associated with lower any-cause mortality. Furthermore TH has the potential to reduce number of days lost to hospitalisation and death. 